\title{
Why being an expert - despite xpert - remains crucial for children in high TB burden settings
}

\author{
Jason M. Bacha ${ }^{1,2^{*}}$, Katherine Ngo ${ }^{3}$, Petra Clowes ${ }^{4}$, Heather R. Draper ${ }^{3}$, Elias N. Ntinginya ${ }^{4}$, Andrew DiNardo $^{3}$, \\ Chacha Mangu ${ }^{4}$, Issa Sabi ${ }^{4}$, Bariki Mtafya ${ }^{4}$ and Anna M. Mandalakas ${ }^{3}$
}

\begin{abstract}
Background: As access to Xpert expands in high TB-burden settings, its performance against clinically diagnosed TB as a reference standard provides important insight as the majority of childhood TB is bacteriologically unconfirmed. We aim to describe the characteristics and outcomes of children with presumptive TB and TB disease, and assess performance of Xpert under programmatic conditions against a clinical diagnosis of TB as a reference standard.

Methods: Retrospective review of children evaluated for presumptive TB in Mbeya, Tanzania. Baseline characteristics were compared by TB disease status and, for patients diagnosed with TB, by TB confirmation status using Wilcoxon rank sum test for continuous variables and the Chi-square test for categorical variables. Sensitivity and specificity were calculated to assess the performance of Xpert, smear, and culture against clinical TB. Kappa statistics were calculated to assess agreement between Xpert and smear to culture.
\end{abstract}

Results: Among children ( $N=455)$ evaluated for presumptive TB, 70.3\% (320/455) had Xpert and 62.8\% (286/455) had culture performed on sputa. 34.5\% (157/455) were diagnosed with TB: 80.3\% (126/157) pulmonary TB, 13.4\% (21/157) bacteriologically confirmed, 53.5\% (84/157) HIV positive, and 48.4\% (76/157) inpatients. Compared to the reference standard of clinical diagnosis, sensitivity of Xpert was 8\% (95\% Cl 4-15), smear 6\% (95\% Cl 3-12) and culture 16\% (95\% Cl 9-24), and did not differ based on patient disposition, nutrition or HIV status.

Conclusion: Despite access to Xpert, the majority of children with presumptive TB were treated based on clinical diagnosis. Reflecting the reality of clinical practice in resource limited settings, new diagnostics such as Xpert serve as important adjunctive tests but will not obviate the need for astute clinicians and comprehensive diagnostic algorithms.

Keywords: Childhood TB, Clinical diagnosis, Diagnostic test performance, Tanzania

\section{Background}

In 2014, one million cases of childhood tuberculosis (TB) resulted in 140,000 pediatric deaths worldwide [1]. Difficulties in diagnosing TB in children, coupled with the risk of rapid, life-threatening progression of untreated disease, make childhood TB a public health crisis in high TB burden settings. Global data suggest that only $35 \%$ of pediatric cases are diagnosed and notified [2]. The true magnitude of childhood TB in high burden settings is

\footnotetext{
* Correspondence: bacha@bcm.edu

'Baylor College of Medicine Children's Foundation - Tanzania, Centre of Excellence at Mbeya Zonal Referral Hospital, Mbeya, Tanzania

2Baylor International Pediatric AIDS Initiative (BIPAI) at Texas Children's

Hospital, Baylor College of Medicine, Houston, TX, USA

Full list of author information is available at the end of the article
}

difficult to ascertain due to under-diagnosis and underreporting [3,4]. Moreover in Tanzania only $23 \%$ of an estimated 270,000 TB cases were notified in 2014, and children comprised just $10 \%$ of these notified cases [1].

Firm diagnosis and confirmation of childhood TB remain challenging due to bacteriologic confirmation by culture, the universally acknowledged reference standard, being suboptimal in children. In children, the sensitivity of culture varies (range 1.5\%-65\%) depending on age, disease severity, number of specimens collected and disease prevalence of the setting [5-8]. The introduction of the Xpert MTB/RIF assay (Xpert; Cepheid, Sunnyvale, CA, USA) brought promise as it showed improved sensitivity, specificity and accuracy compared to smear 
microscopy in culture-positive pediatric cases $[5,9,10]$. Xpert was endorsed by World Health Organization (WHO) as an initial diagnostic test in children, though as a conditional recommendation with very low quality of evidence [11]. However, when evaluated in respiratory samples from culture-negative children started on anti-tuberculosis therapy, Xpert sensitivity was only $2-4 \%$ [ 5 , 11]. Due to the paucibacilllary nature of the disease in children, the majority of childhood TB cases are smear and culture negative, highlighting a potential limitation when applying Xpert in these cases.

Subsequently, even with access to Xpert, the diagnosis of childhood TB in high TB-burden countries such as Tanzania relies upon the clinician's ability to synthesize symptoms, radiographic findings, TB exposure history, and/or immunologic evidence of Mycobacterium tuberculosis infection. The use of a clinical TB diagnosis as a reference standard against which to evaluate the performance of diagnostic tests is an imperfect approach, but it affords an important perspective on test performance in this setting. As Tanzania and other high TBburden countries roll out Xpert technology across expanding levels of care, its performance in programmatic conditions must be continually evaluated to effectively inform policy makers. Upfront Xpert testing in children has shown superior yield over smear microscopy [12, 13], but further assessment in children presenting with a wide variety of disease manifestations in varied settings is needed. In our retrospective descriptive study, we described the characteristics and outcomes of children with presumptive $\mathrm{TB}$ and $\mathrm{TB}$ disease, and assessed the performance of Xpert under programmatic conditions against a clinical diagnosis of TB as a reference standard. The approach was applied among children with a range of disease severity in the context of an outpatient pediatric clinic in Mbeya, Tanzania.

\section{Methods}

\section{Ethical approval}

Approval was obtained from all necessary ethical bodies including the Mbeya Medical Research and Ethics Committee and the National Institute of Medical Research (NIMR) in Tanzania, and the Institutional Review Board, Baylor College of Medicine, Houston, Texas, USA. Waiver of consent was approved by all committees as this retrospective study analyzed only de-identified data.

\section{Study setting}

This retrospective descriptive study was conducted at the Baylor College of Medicine Children's Foundation Tanzania Centre of Excellence (COE) at Mbeya Zonal Referral Hospital (MZRH), the single zonal referral hospital for the Southern Highlands Zone of Tanzania, with a catchment area serving over 3.2 million children aged 0-14 years old [14]. The Southern Highlands Zone reported more than $10 \%$ of Tanzania's notified TB cases [15]. The estimated burden of multidrug-resistant TB in Tanzania is $<1 \%$ [1]. The Mbeya COE is a familycentered, pediatric prevention, care and treatment centre offering comprehensive care to both HIV positive and HIV negative children affected by TB, malnutrition and other chronic conditions. A variety of different cadres, including medical officers, nurses and paediatricians, cared for patients at the COE. All clinicians undergo a one month orientation on comprehensive pediatric care (including childhood TB). The clinical pediatric experience varies among $\mathrm{COE}$ clinicians (e.g. medical officers $0-2$ years; nurses $1-3$ years; pediatricians $2-4$ years). In 2011, in collaboration with the National Institute for Medical Research-Mbeya Medical Research Center (NIMR-MMRC) and MZRH, the COE expanded its pediatric TB services to provide advanced diagnostics (including Xpert) and comprehensive TB treatment initiation and follow up.

\section{Study population and clinical procedures}

Retrospective data were collected on children $(<15$ years of age) evaluated for presumptive TB and/or referred for TB treatment between March 2013 and December 2014. Clinicians used a combination of TB screening questions (e.g. history of known TB contact, failure to gain weight/ weight loss, persistent cough, persistent fever, reduced activities or irritability) and clinical examination findings (e.g. malnutrition, lymphadenopathy, abnormal lung findings), based on national guidelines [16], to identify children with presumptive $\mathrm{TB}$ at the $\mathrm{COE}$ and in the inpatient pediatric wards at MZRH. Presumptive TB patients underwent diagnostics and evaluation at the $\mathrm{COE}$, including sputa analyzed by Xpert, smear, and culture, tuberculin skin testing (TST), chest $\mathrm{x}$-ray (CXR), and/or fine needle aspiration (FNA). Positive TST was defined as TST $\geq 5 \mathrm{~mm}$. Children unable to produce sputum spontaneously completed sputum induction. The NIMR-MMRC TB lab processed sputa samples as previously described [10]. Due to low volumes of collected samples, smear and Xpert were performed routinely on the first sample received, while culture was only performed if a second sample was collected. Digital chest radiography was interpreted by the clinician using the Template Chest Radiography Review Tool [17] to assign the following grades: 'normal', 'abnormal - suspicious for TB' (if evidence of lymphadenopathy, pleural effusion, cavities, miliary patterns, and/or airspace consolidation) and 'abnormal - not TB'. FNA samples were collected in patients with unexplained lymphadenopathy and were sent to MZRH pathology department for histological review. All diagnostic tests were performed prior to or on the day of anti-tuberculosis treatment (ATT) initiation. 
TB diagnosis was based on a combination of clinical history and findings, diagnostic results, and/or microbiological or histological data. Children with confirmed or clinically diagnosed TB were classified into one of three predefined categories of diagnostic certainty (confirmed, probable, possible TB), modeled after the internationallyaccepted consensus definition of intrathoracic childhood TB [17] to also include EPTB (Table 1). Children with alternative explanations for their symptoms and clinical improvement without TB treatment were defined as "Not TB." All patient data were reviewed throughout the 22 month study period and all children who initiated ATT were followed for the full 6 months of treatment to ensure that children diagnosed with and treated for TB had a positive treatment response and lacked an alternative, non-TB explanation of their symptoms, and that children classified as "Not TB" did not later developed TB. Treatment outcomes were based on WHO definitions [18].

\section{Statistical analysis}

Data was extracted using a standardized data collection tool and analyzed using Stata 12.1 SE software (StataCorp2011, College Station, TX, USA). Baseline patient characteristics were summarized using descriptive statistics and compared by TB status (TB disease vs not TB disease). For patients diagnosed with TB, baseline patient characteristics were summarized using descriptive

Table 1 Diagnostic certainty categories for cases of TB disease in children ${ }^{a}$

\begin{tabular}{|c|c|}
\hline Diagnostic Certainty Group & Definition of Case Categories \\
\hline Confirmed tuberculosis & $\begin{array}{l}\text { A child with Mycobacterium tuberculosis } \\
\text { identified from a clinical specimen using } \\
\text { any microbiologic diagnostic test available } \\
\text { (e.g. smear, culture, or Xpert) and at least } \\
\text { one sign or symptom suggestive of } \\
\text { tuberculosis }\end{array}$ \\
\hline Probable tuberculosis & $\begin{array}{l}\text { A child with one or more of the following } \\
\text { clinical symptoms: } \\
\text { - } \quad \text { Abnormal CXR consistent with TB } \\
\text { - Cough duration greater than } 2 \text { weeks } \\
\text { - Weight loss or failure to thrive } \\
\text { (WHZ-score less than -2) } \\
\text { - Fever greater than } 2 \text { weeks } \\
\text { - } \quad \text { Signs/symptoms of disseminated TB } \\
\text { And one (or more) of the following: } \\
\text { - Documented exposure to TB in the } \\
\text { - } \text { preceding } 24 \text { months } \\
\text { tuberculosis treatment } \\
\text { - Immunologic (TST or IGRA) evidence of } \\
\text { Mycobacterium tuberculosis infection }\end{array}$ \\
\hline Possible tuberculosis & $\begin{array}{l}\text { A child with one or more of the following } \\
\text { clinical symptoms: } \\
\text { - Abnormal CXR consistent with TB } \\
\text { - Cough greater than } 2 \text { weeks } \\
\text { - Weight loss or failure to thrive } \\
\text { (WHZ-score less than -2) } \\
\text { - Fever greater than } 2 \text { weeks }\end{array}$ \\
\hline
\end{tabular}

${ }^{\mathrm{a} A d a p t e d ~ f r o m ~ G r a h a m ~ e t ~ a l . ~[17] ~}$ statistics and compared by confirmation status (bacteriologically confirmed vs clinically diagnosed). Age was analyzed using the Wilcoxon rank sum test, and all other categorical baseline measures were analysed using the Chi-square test to determine if these characteristics were associated with TB disease or confirmation status as appropriate. Children diagnosed with TB disease - either confirmed, possible or probable TB - were classified as "clinical TB", which served as our primary reference standard (and incorporated both CXR and TST results). Sensitivity and specificity were calculated to assess the performance of Xpert, smear, and culture against clinical TB. Test performances were analyzed for all children and subgroups. Patients who did not have diagnostic tests performed or did not have recorded results (e.g. error/invalid) were excluded from calculations. The Kappa statistic, overall agreement, positive agreement and negative agreement were calculated to assess agreement between the three tests [19] for patients who had a positive or negative test result for all three tests.

\section{Results}

Between March 2013 and December 2014, 455 children with presumptive TB were evaluated. Of those, 157 (34.5\%) were diagnosed with TB. Baseline characteristics and diagnostic tests performed among those diagnosed with and without TB did not differ, with the exception of FNA being performed more often in those with TB compared to without $[8.3 \%(13 / 157)$ vs $3.7 \%(11 / 298), p=0.035$; Table 2]. Final diagnostic certainty categories were $13.4 \%(21 / 157)$ bacteriologically confirmed TB, $63.1 \%$ (99/157) probable TB, and 23.6\% (37/157) possible TB (Table 3). The majority of children had pulmonary TB $(80.3 \%, 126 / 157)$. Bacteriologically confirmed TB cases were older (median age 9.4 years vs 4.6 years, $p=0.017$ ) and more likely to provide sputum for analysis [100\% (21/21) vs $69.1 \%(94 / 136), p=0.001]$ compared to clinically diagnosed TB cases (Table 4).

Ninety-nine percent $(155 / 157)$ of children with a TB diagnosis were initiated on ATT, with one child dying and one child lost-to-follow up (LTFU) before treatment could be initiated. Sixty-nine percent of children (109/ 157) showed treatment success, $7.0 \%(11 / 157)$ were not evaluated/transferred out, 7.6\% (12/157) died and 15.9\% (25/157) were LTFU (Table 3). All children followed through the completion of their treatment showed positive clinical response to TB treatment for the duration of their follow up.

Among those producing an initial sputum $(n=320)$, $2.8 \%$ of children $(9 / 320,95 \%$ CI $1.3-5.3)$ had a positive Xpert and 2.2\% of children (7/320, 95\% CI 0.9-4.5) had a positive smear (Figure 1). Culture was positive in 5.6\% (16/286, 95\% CI 3.2-8.9) of those producing a second sputum. Xpert was positive in $7.1 \%(4 / 56,95 \%$ CI $2.0-$ 
Table 2 Baseline characteristics of patients referred for presumptive TB $(N=455)$

\begin{tabular}{llll}
\hline & TB disease ${ }^{\mathrm{a}}(n=157)$ & Not TB disease $(n=298)$ & $p$-value \\
\hline Median age in years at time of TB referral (IQR) & $5.0(1.6-9.5)$ & $4.4(1.5-10.0)$ & 0.872 \\
Male gender (\%) & $75(47.8)$ & $139(46.6)$ & 0.819 \\
HIV positive (\%) & $84(53.5)$ & $163(54.7)$ & 0.808 \\
Disposition at time of referral of "Inpatient" (\%) & $76(48.4)$ & $9(3.0)$ & 0.878 \\
Past TB treatment (\%) & $7(4.5)$ & $119(39.9)$ & 0.428 \\
Severe Malnutrition (\%) & $50(31.9)$ & $52(17.5)$ & 0.090 \\
Reported TB contact (\%) & $33(21.0)$ & & 0.353 \\
Diagnostic Tests Performed & & $205(68.8)$ & $176(59.1)$ \\
$\quad$ Sputum analysis (\%) & $115(73.3)$ & $264(88.6)$ & 0.323 \\
Chest x-ray (\%) & $107(68.2)$ & $11(3.7)$ & 0.057 \\
Tuberculin skin test (\%) & $143(91.1)$ & 0.411 \\
Fine needle aspirate (\%) & $13(8.3)$ & 0.037 \\
\hline
\end{tabular}

${ }^{\mathrm{a}} \mathrm{TB}$ disease includes all children meeting criteria for confirmed, possible or probable TB. ${ }^{\mathrm{b}}$ Severe acute malnutrition is defined as weight-for-height $\mathrm{Z}$ score $<3 \mathrm{SD}$ and/or presence of edema [29]

Table 3 Characteristics and treatment outcomes of children diagnosed with TB $(N=157)$

\begin{tabular}{|c|c|c|}
\hline Characteristic & Number & $\%$ \\
\hline \multicolumn{3}{|l|}{ Treatment Initiation } \\
\hline ATT initiated & 155 & $998.7 \%$ \\
\hline $\begin{array}{l}\text { Median time from referral to initiation of } \\
\text { ATT (days, IQR) }\end{array}$ & 3 days & $1.00-6.25$ days \\
\hline \multicolumn{3}{|l|}{ Diagnostic Certainty } \\
\hline Confirmed TB & 21 & $13.4 \%$ \\
\hline Confirmed pulmonary TB & 20 & $95.2 \%$ \\
\hline Confirmed EPTB & 1 & $4.8 \%$ \\
\hline Confirmed LNTB & 0 & $0.0 \%$ \\
\hline Probable TB & 99 & $63.1 \%$ \\
\hline Possible TB & 37 & $23.6 \%$ \\
\hline \multicolumn{3}{|l|}{ Type of TB } \\
\hline Pulmonary TB & 126 & $80.3 \%$ \\
\hline EPTB (excluding lymph node TB) & 19 & $12.1 \%$ \\
\hline Lymph node TB & 12 & $7.6 \%$ \\
\hline \multicolumn{3}{|l|}{ Treatment Outcomes } \\
\hline Cured $^{\mathrm{a}}$ & 19 & $12.1 \%$ \\
\hline Treatment Completed ${ }^{\mathrm{b}}$ & 90 & $57.3 \%$ \\
\hline Transferred Out/Not Evaluated & 11 & $7.0 \%$ \\
\hline Died & 12 & $7.6 \%$ \\
\hline Lost-to-follow up & 25 & $15.9 \%$ \\
\hline
\end{tabular}

Abbreviations: $A T T$, anti-tuberculosis therapy; EPTB, extrapulmonary TB; LNTB, lymph node TB

${ }^{a}$ Cured $=$ bacteriologically confirmed TB at the beginning of treatment who was smear- or culture-negative in the last month of treatment and on at least one previous occasion. ${ }^{\mathrm{b}}$ Treatment Completed $=$ clinically diagnosed or bacteriologically confirmed TB patient who completed treatment without evidence of failure, but with no record of sputum smear or culture results in the last month of treatment were negative, either because tests were not done or because no results available [18]
17.3) of the no sample for culture TB cases and none $(0 / 85,95 \%$ CI $0.0-4.2)$ of the culture negative TB cases. No rifampicin resistance was detected by Xpert.

When compared to a reference standard of clinical diagnosis, the sensitivities of culture, Xpert and smear were $16 \%$ (95\% CI 9-24), $8 \%$ (95\% CI 4-15), and 6\% (95\% CI 3-12), respectively. The sensitivity of culture, Xpert and smear did not significantly vary by inpatient versus outpatient disposition or by HIV-status (Figure 2). Specificity of culture, Xpert, and smear was high in all groups when using 'clinical TB' as reference standard (range of 0.99-1.00, 95\% CIs 0.95-1.00). Kappa statistics for level of agreement demonstrated higher agreement for Xpert and culture compared to agreement for smear and culture in all patients and subgroups with the exception of HIV negative children (Table 5; see Additional File 1 for comparison of Xpert and clinical TB to the culture as reference standard).

TST and CXR was performed in $89.5 \%$ (407/455) and $62.2 \%(283 / 455)$, respectively, of children with presumptive TB. Overall, $13.5 \%$ (55/407) of TSTs were positive and $23.3 \%(66 / 283)$ of CXR were resulted as 'abnormal suspicious for TB' (see Additional File 2 for positivity rates of TST and CXR among subgroups). HIV positive children were more likely to have sputum analysis $(76.6 \%$ vs $62.5 \%, \mathrm{p}=0.001)$ and less likely to have TST done $(86.6 \%$ vs $92.8 \%, p=0.03)$, compared to HIV negative children. There were no differences in CXR or FNA performed between the two groups.

\section{Discussion}

Our study demonstrated that among Tanzanian children receiving care at a pediatric clinic in a resourceconstrained setting, the yield of Xpert against a clinical diagnosis of $\mathrm{TB}$ was limited. When compared to this 
Table 4 Baseline characteristics of clinically diagnosed versus bacteriologically confirmed TB patients $(N=157)$

\begin{tabular}{|c|c|c|c|}
\hline & $\begin{array}{l}\text { Clinically diagnosed TB (possible + probable TB) } \\
(n=136)\end{array}$ & $\begin{array}{l}\text { Bacteriologically confirmed } \text { TB }^{\text {a }} \\
(n=21)\end{array}$ & $p$-value \\
\hline Median age in years at time of TB referral (IQR) & $4.6(1.5-9.1)$ & $9.4(4.5-12.1)$ & 0.017 \\
\hline Male gender (\%) & $68(50.0)$ & $14(66.7)$ & 0.155 \\
\hline HIV positive (\%) & $73(53.7)$ & $11(52.4)$ & 0.912 \\
\hline Disposition at time of referral of "Inpatient" (\%) & $69(50.7)$ & $7(33.3)$ & 0.137 \\
\hline Past TB treatment (\%) & $5(3.7)$ & $2(9.5)$ & 0.237 \\
\hline Severe Malnutrition (\%) & $42(30.9)$ & $8(38.1)$ & 0.509 \\
\hline Reported TB contact (\%) & $28(20.6)$ & $5(23.8)$ & 0.736 \\
\hline \multicolumn{4}{|l|}{ Diagnostic Tests Performed } \\
\hline Sputum analysis (\%) & $94(69.1)$ & $21(100.0)$ & 0.001 \\
\hline Chest x-ray (\%) & $91(66.9)$ & $16(76.2)$ & 0.460 \\
\hline Tuberculin skin test (\%) & $123(90.1)$ & $20(95.2)$ & 0.695 \\
\hline Fine needle aspirate (\%) & $13(100.0)$ & $0(0.0)$ & 0.218 \\
\hline
\end{tabular}

${ }^{\mathrm{a} B}$ Bacteriologically confirmed TB is a TB case from whom a biological specimen is positive by smear microscopy, culture or Xpert MTB/RIF. ${ }^{\mathrm{b}} \mathrm{Severe}$ acute malnutrition is defined as weight-for-height $Z$ score $<3$ SD and/or presence of edema [29]

reference standard, the sensitivity of Xpert was $8 \%$. Although marginally better than smear ( $6 \%$ sensitivity), Xpert was half as sensitive as culture (16\% sensitivity). Large proportions of children with presumptive TB presented in a severely compromised state with multiple comorbidities (54.3\% HIV positive, 47.9\% hospitalized, $37.1 \%$ severely malnourished). This likely lowered clinicians' thresholds for empiric TB treatment and contributed to the high numbers of clinical TB diagnoses (86.7\% of TB cases). Our findings demonstrate that even with access to Xpert, TB treatment decisions rely heavily on clinical diagnosis, particularly in critically ill children.

The effect and interplay between Xpert and empiric TB treatment remains complex [20], and our findings highlight the limitations of Xpert when used in children diagnosed with culture-negative (or not performed) TB in

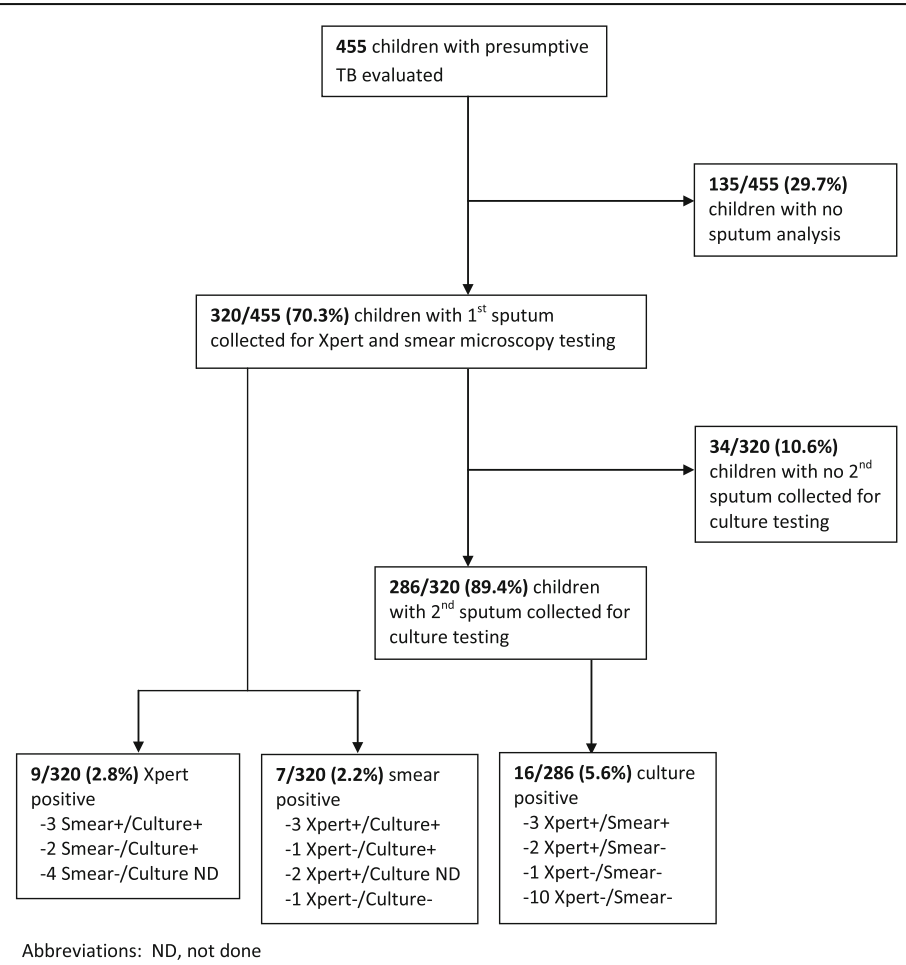

Fig. 1 Sputum analyses performed and sputum results for children with presumptive TB 


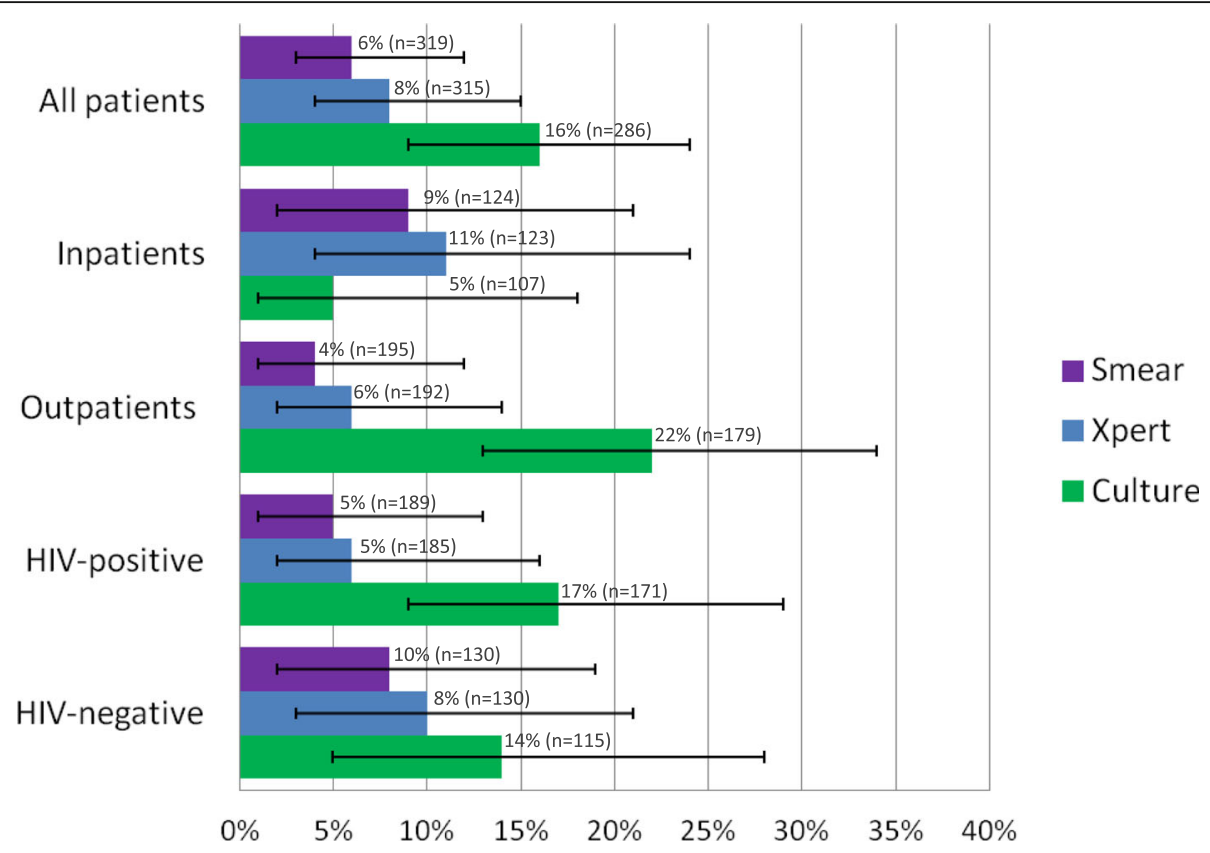

Fig. 2 Sensitivities of smear, Xpert and culture among all patients and subgroups using "clinical TB" as the primary reference standard (with error bars representing $95 \% \mathrm{Cls}$ )

a high burden setting. Our estimates of Xpert sensitivity are consistent with previous research comparing the performance of Xpert to clinical diagnosis in culture-negative pediatric cases $[5,21,22]$, as well as an earlier prospective study in our same study setting [10] that demonstrated $8.5 \%$ sensitivity of Xpert in children clinically diagnosed with TB. While that study evaluated three sputa samples for every patient, our programmatic approach allowed a maximum of one sputum sample for culture. The use of multiple sputum samples improves Xpert test performance $[10,23]$, however this approach is not feasible in our, nor in many other, resource-constrained settings. A thoughtful comparison of these differences further highlights the reality and challenges of TB diagnostics, care and treatment of children presenting in the clinical care arena rather than a research setting.

Emerging evidence suggests under-diagnosis of TB may be common in children with severe acute malnutrition
(SAM) and/or HIV and their mortality is high [7, 24, 25]. We were able to evaluate TB diseased children who presented with a wide spectrum of disease severity and comorbidities - including HIV co-infection, SAM, and inpatient disposition - and were unable to show a difference in performance of Xpert in children based on their HIV, malnutrition, or inpatient status. We postulate that in these children clinicians have lower thresholds for empiric TB treatment, and their TB infection may have progressed more rapidly to severe disease, despite lower, undetectable mycobacterial loads.

The impact of any new diagnostic test - such as Xpert - depends greatly on how it is implemented outside of research settings and on the context in which it is implemented [26]. Two recent randomized controlled trials evaluating the impact of Xpert in adults in high TB burden settings showed that despite increased sensitivity and decreased turnaround time, its use did not translate

Table 5 Agreement and discordance of culture versus Xpert and smear stratified by referral type and HIV status

\begin{tabular}{|c|c|c|c|c|c|c|c|c|}
\hline & \multicolumn{4}{|c|}{ Culture versus Xpert MTB/RIF results } & \multicolumn{4}{|c|}{ Culture versus smear results } \\
\hline & $\begin{array}{l}\text { no. with agreement/ } \\
\text { no. tested }\end{array}$ & $\begin{array}{l}\text { PA (\%)/ } \\
\text { NA (\%) }\end{array}$ & $\begin{array}{l}\text { Overall } \\
\text { Agreement (\%) }\end{array}$ & $\begin{array}{l}\text { Kappa } \\
(95 \% \mathrm{Cl})\end{array}$ & $\begin{array}{l}\text { no. with agreement/ } \\
\text { no. tested }\end{array}$ & $\begin{array}{l}\text { Overall } \\
\text { Agreement (\%) }\end{array}$ & $\begin{array}{l}\text { PA (\%)/ } \\
\text { NA (\%) }\end{array}$ & $\begin{array}{l}\text { Kappa } \\
(95 \% \text { Cl) }\end{array}$ \\
\hline All children & $270 / 282$ & $45.5 / 97.8$ & 95.7 & $0.44(0.18-0.70)$ & $268 / 282$ & 95.0 & $36.4 / 97.4$ & $0.35(0.09-0.60)$ \\
\hline Inpatient & 105/106 & $66.7 / 99.5$ & 99.1 & $0.66(0.04-1.00)$ & $104 / 106$ & 98.1 & $50.0 / 99.0$ & $0.49(-0.12-1.00)$ \\
\hline Outpatient & $165 / 176$ & $42.1 / 96.7$ & 93.8 & $0.40(0.13-0.67)$ & $164 / 176$ & 93.2 & $33.3 / 96.4$ & $0.31(0.04-0.59)$ \\
\hline HIV positive & $160 / 167$ & $53.3 / 97.8$ & 95.8 & $0.52(0.21-0.82)$ & $157 / 167$ & 94.0 & 28.6/96.9 & $0.27(-0.04-0.57)$ \\
\hline HIV negative & $110 / 115$ & 28.6/97.8 & 95.7 & $0.28(-0.15-0.70)$ & $111 / 115$ & 96.5 & $50.0 / 98.2$ & $0.49(0.06-0.91)$ \\
\hline
\end{tabular}


into patient important outcomes such as reduced morbidity or mortality $[27,28]$. Xpert is particularly useful when positive in pediatric cases where a clinical diagnosis is unclear. However, a negative Xpert result cannot exclude the possibility of TB disease, especially in children living in areas of high TB burden. In our setting, the majority of childhood TB cases were still diagnosed clinically, demonstrating how, even though Xpert is a valuable additional test in children, it is not a standalone replacement to culture and clinical diagnosis. Clinicians must be made aware of these limitations and resources should be utilized to strengthen all components of the diagnostic algorithm in concert.

Our study was limited by its retrospective approach assessing TB care at a single site. Despite clinical practice following national guidelines, there was variability in the diagnostic tests performed per patient, reflecting the reality of clinical practice in $\mathrm{TB}$ high burden, resource constrained settings. This variability provided unique and realistic insights regarding the incorporation of new diagnostics into clinical care. Our site received many referrals, contributing to potential referral bias that lowered clinicians' thresholds to treat empirically. High levels of empiric treatment may limit the performance of Xpert against clinical practice. Although there were few bacteriologically confirmed cases, our study benefited from long follow up of at least six months of all patients to ensure that clinically diagnosed TB cases improved on ATT and 'not TB' cases improved without ATT. Our study did not specifically look at impact of Xpert on clinician behavior or favorable patient outcomes, but provided the basic foundation for such future research. Overall, these findings emphasize the importance of evaluating new diagnostics in children against clinical diagnosis as reference standards.

\section{Conclusion}

Despite access to Xpert technology, the majority of childhood TB cases presenting to a pediatric referral centre in a resource limited setting were diagnosed clinically, thus limiting the contribution of Xpert to clinical decision making under our programmatic conditions. Recognizing the paucibacillary nature of childhood TB and high risk of rapid disease progression in children, new diagnostics such as Xpert serve as important adjunctive tests but will not obviate the need for astute clinicians and comprehensive diagnostic algorithms. Care should be taken to carefully balance allocation of scarce resources between new diagnostic technologies (e.g. Xpert) and educational efforts to foster traditional components of the diagnostic algorithm (e.g. history, physical exam, radiography, TST) for childhood TB in resource limited settings.

\section{Additional files}

Additional file 1: Performance outcomes of clinical TB diagnosis and Xpert MTB/RIF using culture as reference standard for patients $<15$ years old and referred for presumptive TB. The data in additional file 1 shows the sensitivity, specificity, PPV and NPV of clinical TB and Xpert MTB/RIF compared to culture as the reference standard for all patients as well as the subgroups of inpatients, outpatients, HIV positive and HIV negative. (DOCX $12 \mathrm{~kb}$ )

Additional file 2: Tuberculin Skin Test (TST) and chest x-ray positivity rates among children with presumptive TB. The data in additional file 2 shows the positivity rates of TST and CXR for subgroups of presumptive TB, TB disease, HIV positive, HIV negative, inpatients and outpatients. (DOCX $11 \mathrm{~kb})$

\section{Acknowledgements}

We would like to thank the staff at the Baylor College of Medicine Children's Foundation - Tanzania Centres of Excellence and the NIMR-MMRC TB laboratory for their tireless work and dedication towards improving the lives of our patients and their families. We also wish to acknowledge the courage, strength and resilience of our patients and their families as they overcome their battles with TB.

\section{Funding}

This manuscript resulted (in part) from research supported by the Baylor-UTHouston Center for AIDS Research (CFAR), an NIH funded program (Al036211).

\section{Availability of data and materials}

The datasets used and/or analysed during the current study are available from the corresponding author on reasonable request.

\section{Authors' contributions}

JB was primarily responsible for clinical aspects of the study program, and $P C$ and BM were primarily responsible for the TB lab aspects of the study program. JB, KN, HD and AM were responsible for design of program, data collection, statistical analysis and writing of the manuscript. All authors contributed to the plan of analysis, interpretation of the data, commenting on manuscript drafts and approval of the final version. All authors read and approved the final manuscript.

\section{Competing interests}

Anna M. Mandalakas is a member of the editorial board of BMC Infectious Diseases. She was not involved in the review of this manuscript. The authors have no other competing interests to declare.

\section{Consent for publication}

Not applicable.

\section{Ethics approval and consent to participate}

Approval was obtained from all necessary ethical bodies including the Mbeya Medical Research and Ethics Committee and the National Institute of Medical Research (NIMR) in Tanzania, and the Institutional Review Board, Baylor College of Medicine, Houston, Texas, USA. Waiver of consent was approved by all committees as this retrospective study analyzed only de-identified data.

\section{Author details}

${ }^{1}$ Baylor College of Medicine Children's Foundation - Tanzania, Centre of Excellence at Mbeya Zonal Referral Hospital, Mbeya, Tanzania. ${ }^{2}$ Baylor International Pediatric AIDS Initiative (BIPAI) at Texas Children's Hospital, Baylor College of Medicine, Houston, TX, USA. ${ }^{3}$ The Global Tuberculosis Program, Texas Children's Hospital, Global and Immigrant Health, Department of Pediatrics Baylor College of Medicine, Houston, TX 77030, USA. ${ }^{4}$ National Institute of Medical Research-Mbeya Medical Research Centre, Mbeya, Tanzania. 
Received: 1 October 2016 Accepted: 31 January 2017

Published online: 06 February 2017

\section{References}

1. World Health Organization. Global tuberculosis report. Geneva: World Health Organization; 2015.

2. Dodd PJ, Gardiner E, Coghlan R, Seddon JA. Burden of childhood tuberculosis in 22 high-burden countries: a mathematical modelling study. Lancet Glob Health. 2014;2(8):e453-9.

3. Coghlan R, Gardiner E, Amanullah F, Ihekweazu C, Triasih R, Grzemska M, et al. Understanding market size and reporting gaps for paediatric TB in Indonesia, Nigeria and Pakistan: supporting improved treatment of childhood TB in the advent of new medicines. PLoS One. 2015;10(10):e0138323.

4. Lestari T, Probandari A, Hurtig AK, Utarini A. High caseload of childhood tuberculosis in hospitals on java island. Indonesia: a cross sectional study. BMC Public Health. 2011;11:784

5. Detjen AK, DiNardo AR, Leyden J, Steingart KR, Menzies D, Schiller I, et al. Xpert MTB/RIF assay for the diagnosis of pulmonary tuberculosis in children: a systematic review and meta-analysis. Lancet Respir Med. 2015;3(6):451-61.

6. Graham SM, Cuevas LE, Jean-Philippe P, Browning R, Casenghi M, Detjen AK, et al. Clinical case definitions for classification of intrathoracic tuberculosis in children: an update. Clin Infect Dis. 2015;61 Suppl(3):S179-87.

7. LaCourse SM, Chester FM, Preidis G, McCrary LM, Arscott-Mills T, Maliwichi $\mathrm{M}$, et al. Use of xpert for the diagnosis of pulmonary tuberculosis in severely malnourished hospitalized Malawian children. Pediatr Infect Dis J. 2014:33(11):1200-2.

8. Nhu NT, Ha DT, Anh ND, Thu DD, Duong TN, Quang ND, et al. Evaluation of xpert MTB/RIF and MODS assay for the diagnosis of pediatric tuberculosis. BMC Infect Dis. 2013;13:31.

9. Nicol MP, Whitelaw A, Wendy S. Using xpert MTB/RIF. Curr Respir Med Rev. 2013;9:187-92.

10. Rachow A, Clowes P, Saathoff E, Mtafya B, Michael E, Ntinginya EN, et al. Increased and expedited case detection by xpert MTB/RIF assay in childhood tuberculosis: a prospective cohort study. Clin Infect Dis. 2012;54(10):1388-96

11. World Health Organization. WHO Guidelines Approved by the Guidelines Review Committee. Automated Real-Time Nucleic Acid Amplification Technology for Rapid and Simultaneous Detection of Tuberculosis and Rifampicin Resistance: Xpert MTB/RIF Assay for the Diagnosis of Pulmonary and Extrapulmonary TB in Adults and Children: Policy Update. Geneva: World Health Organization; 2013

12. Raizada N, Sachdeva KS, Nair SA, Kulsange S, Gupta RS, Thakur R, et al. Enhancing TB case detection: experience in offering upfront xpert MTB/RIF testing to pediatric presumptive TB and DR TB cases for early rapid diagnosis of drug sensitive and drug resistant TB. PLoS One. 2014;9(8): e105346.

13. Raizada N, Sachdeva KS, Swaminathan S, Kulsange S, Khaparde SD, Nair SA, et al. Piloting upfront xpert MTB/RIF testing on various specimens under programmatic conditions for diagnosis of TB \& DR-TB in paediatric population. PLoS One. 2015;10(10):e0140375

14. Tanzanian National Bureau of Statistics (NBS) and Office of Chief Government Statistician (OCGS). The 2012 population and housing census: basic demographic and socio-economic profile; Key findings. 2016. Dar es Salaam: NBS and OCGS; 2014.

15. National Tuberculosis and Leprosy Programme. Manual for the management of tuberculosis and leprosy. 6th ed. Dar es Salaam: United Republic of Tanzania Ministry of Health and Social Welfare; 2013.

16. National Tuberculosis and Leprosy Programme. National guidelines for the management of tuberculosis in children. 1st ed. Dar es Salaam: The United Reublic of Tanzania Ministry of Health and Social Welfare; 2012.

17. Graham SM, Ahmed T, Amanullah F, Browning R, Cardenas V, Casenghi M, et al. Evaluation of tuberculosis diagnostics in children: 1. Proposed clinical case definitions for classification of intrathoracic tuberculosis disease. Consensus from an expert panel. J Infect Dis. 2012;205 Suppl(2):S199-208.

18. World Health Organization. Definitions and reporting framework for tuberculosis - 2013 revision. Geneva: WHO Press; 2013. Updated December 2014.

19. Cicchetti DV, Feinstein AR. High agreement but low kappa: II. Resolving the paradoxes. J Clin Epidemiol. 1990:43(6):551-8.

20. Theron G, Peter J, Dowdy D, Langley I, Squire SB, Dheda K. Do high rates of empirical treatment undermine the potential effect of new diagnostic tests for tuberculosis in high-burden settings? Lancet Infect Dis. 2014;14(6):527-32.
21. Giang Do C, Duong TN, Ha DT, Nhan HT, Wolbers M, Nhu NT, et al. Prospective evaluation of GeneXpert for the diagnosis of HIV- negative pediatric TB cases. BMC Infect Dis. 2015;15:70.

22. Reither K, Manyama C, Clowes P, Rachow A, Mapamba D, Steiner A, et al. Xpert MTB/RIF assay for diagnosis of pulmonary tuberculosis in children: a prospective, multi-centre evaluation. J Infect. 2015;70(4):392-9.

23. Oberhelman RA, Soto-Castellares G, Gilman RH, Caviedes L, Castillo ME, Kolevic $L$, et al. Diagnostic approaches for paediatric tuberculosis by use of different specimen types, culture methods, and PCR: a prospective casecontrol study. Lancet Infect Dis. 2010;10(9):612-20.

24. Chisti MJ, Graham SM, Duke T, Ahmed T, Ashraf H, Faruque AS, et al. A prospective study of the prevalence of tuberculosis and bacteraemia in Bangladeshi children with severe malnutrition and pneumonia including an evaluation of xpert MTB/RIF assay. PLoS One. 2014;9(4):e93776.

25. Chisti MJ, Ahmed T, Pietroni MA, Faruque AS, Ashraf H, Bardhan PK, et al. Pulmonary tuberculosis in severely-malnourished or HIV-infected children with pneumonia: a review. J Health Popul Nutr. 2013;31(3):308-13.

26. Schumacher SG, Thangakunam B, Denkinger CM, Oliver AA, Shakti KB, Qin ZZ, et al. Impact of point-of-care implementation of xpert MTB/RIF: product vs. Process innovation. Int J Tuberc Lung Dis. 2015;19(9):1084-90.

27. Mupfumi L, Makamure B, Chirehwa M, Sagonda T, Zinyowera S, Mason P, et al. Impact of xpert MTB/RIF on antiretroviral therapy-associated tuberculosis and mortality: a pragmatic randomized controlled trial. Open Forum Infect Dis. 2014;1(1):ofu038.

28. Theron G, Zijenah L, Chanda D, Clowes P, Rachow A, Lesosky M, et al. Feasibility, accuracy, and clinical effect of point-of-care xpert MTB/RIF testing for tuberculosis in primary-care settings in africa: a multicentre, randomised, controlled trial. Lancet. 2014;383(9915):424-35.

29. WHO Guidelines Approved by the Guidelines Review Committee. WHO child growth standards and the identification of severe acute malnutrition in infants and children: a joint statement by the world health organization and the united nations Children's fund. Geneva: World Health Organization; 2009.

\section{Submit your next manuscript to BioMed Central and we will help you at every step:}

- We accept pre-submission inquiries

- Our selector tool helps you to find the most relevant journal

- We provide round the clock customer support

- Convenient online submission

- Thorough peer review

- Inclusion in PubMed and all major indexing services

- Maximum visibility for your research

Submit your manuscript at www.biomedcentral.com/submit
Biomed Central 